\title{
SHEAR BOND STRENGTH OF A CONTEMPORARY DENTALADHESIVE SYSTEM TO TWO TYPES OF ZIRCONIA
}

\author{
Heba Mohamed Ahmed Eldawy ${ }^{1}$, Mosaad Aly El-Gabrouny ${ }^{2}$, Diaa Eldin Saad Awad ${ }^{3}$, \\ Amr Abd El-Aziz Shebl ${ }^{4}$
}

DOI: $10.21608 /$ dsu.2020.16570.1012

Manuscript ID: DSU-1909-1012

\section{KEYWORDS}

Adhesive,

Panavia F2.0,

RelyX Ultimate,

Sandblasting ,

Single Bond Universal

Zirconia.
- E-mail address: heba_3776@hotmail.com

1. B.D.S. Suez-Canal University (2006)

2. Professor in Crown and Bridge Department, Faculty of Dentistry, Suez-Canal University

3. Lecturer in Crown and Bridge Department, Faculty of Dentistry, Suez-Canal University

4. Lecturer in Crown and Bridge Department, Faculty of Dentistry, Suez-Canal University

\section{ABSTRACT}

Aim: to evaluate the shear bond strength of an adhesive system (Single bond universal adhesive+ RelyX Ultimate cement) and Panavia F2.0 to two types of zirconia (conventional ZI zirconia and translucent TZI zirconia) after thermocycling. Materials \& Methods: 10 square shaped large samples from Incoris ZI blocks, and 10 from Incoris TZI blocks were prepared with larger dimensions $(12 \times 12 \times 2 \mathrm{~mm})$. Another 10 square shaped small samples from each type of zirconia blocks were with small dimensions $(6 \times 6 \times 2 \mathrm{~mm})$. The surfaces of the large and small samples were air abraded with $110 \mu \mathrm{m}$ AL2O3 particles, then each small sample was bonded to the large sample using two types of cements (RelyX Ultimate cement in combination with Single bond universal adhesive and Panavia F2.0 cement) under $3 \mathrm{Kg}$ load and light cured. After bonding, all specimens were thermocycled for 5000 cycles. The cement/ zirconia interface for each subgroup was detected under scanning electron microscope and the shear bond strength was tested using a computerized universal testing machine. Results: No significant difference between shear bond strength of all subgroups tested. Conclusion: The shear bond strength of RelyX Ultimate+ Single bond universal adhesive to two types of zirconia was comparable to Panavia F2.0, and the shear bond strength of translucent zirconia bonded with two types of cements was comparable to conventional zirconia.

\section{INTRODUCTION}

Many patients are more interested in having esthetic appearance than any other feature of dental service. So, the popularity of all-ceramic restorations has increased in recent years as it offers excellent esthetics and biocompatibility properties ${ }^{(1)}$. They transmitted the light better than any other restorations, which lead to improve reproduction of the color and translucency of natural teeth ${ }^{(2)}$.

Zirconia has been widely used in the field of dentistry because of its high mechanical properties, but has low translucency ${ }^{(3)}$. To overcome the problem of opacity, used a veneer layer of feldspathic ceramic, and achieving a more natural appearance ${ }^{(4)}$.

In recent years, the monolithic zirconia (fully anatomic zirconia or translucent zirconia) have been developed to overcome chipping of the 
veneer ceramic, it needs less preparation because the thickness for restoration is less critical than that of the other ceramic materials ${ }^{(5)}$.

Various methods have been used to improve bonding between zirconia and dental resin cement such as sandblasting, grinding, tribochemical silica coating, laser treatment, hot etching, and selective infiltration-etching (SIE) ${ }^{(6)}$. Mechanically, airborne-particle abrasion has been used to increase surface roughness that allow the resin cement to flow into these micro-retentions and creates a stronger micromechanical interlock, clean the surface, removes impurities, and modify the surface energy and the wettability ${ }^{(7)}$. In addition, airborne-particle abrasion provides the mechanical impingement of particles on the surface,. Resin cements containing phosphate monomer (MDP, 10 methacryloyloxydecyl dihydrogen phosphate) has been used for chemical bonding. Combining mechanical and chemical surface pre-treatments of zirconia provides a durable bond strength ${ }^{(8)}$.

The purpose of the current study was to evaluate the shear bond strength of air-abraded conventional and translucent zirconia with a new adhesive system.

\section{MATERIALS AND METHODS}

10 square shaped large samples from Incoris ZI blocks (C) , and another 10 square shaped large samples from Incoris TZI blocks ( $\mathrm{T}$ ) were cut using low speed cutting saw with larger dimensions (each of $15 \mathrm{~mm} \times 15 \mathrm{~mm}$ dimensions and $2.5 \mathrm{~mm}$ thickness) to compensate sintering shrinkage. Ten square shaped small samples from each type of zirconia blocks were cut by the same method with $7.5 \mathrm{~mm}$ $\mathrm{x} 7.5 \mathrm{~mm}$ dimensions and $2.5 \mathrm{~mm}$ thickness.

All samples were cleaned in distilled water to remove grinding dust, then dried and sintered in a high temperature furnace at temperature $1500^{\circ} \mathrm{C}$ for 7 hours according to the manufacturer's instructions. The final dimensions, after sintering were approximately $12 \mathrm{~mm} \times 12 \mathrm{~mm} \times 2 \mathrm{~mm}$ for the large samples and $6 \mathrm{~mm} \times 6 \mathrm{~mm} \times 2 \mathrm{~mm}$ for the small samples (Figure 1). All fully sintered large samples were embedded in self cured acrylic resin blocks in a rectangular plastic mould with only one side was exposed and would be parallel to the edge of resin blocks and to long axis of the chisel of the universal testing machine during the shear test. A perforated plastic sheet was applied in a rectangular plastic mould and placed on a glass surface. The sample was embedded in the perforated sheet such that it contacted the glass surface and the self cured acrylic resin was mixed. The resin was poured inside the mould over the sample and left until complete setting, then the block was removed from the mould with the large sample embedded in the resin block. All zirconia surfaces of the large and small samples to be bonded were air abraded in sandblasting unit by $110 \mu \mathrm{m} \mathrm{AL}_{2} \mathrm{O}_{3}$ particles under 2.5 bar pressure at a distance $10 \mathrm{~mm}$ for 15 seconds, and samples were ultrasonic cleaned for 10 minutes to remove blasting particles, then air-dried.

\section{A. Panavia F2.0 cement}

Five small conventional zirconia (C) samples were bonded to five large $\mathrm{C}$ samples, and five small translucent zirconia (T) samples were bonded to five large $\mathrm{T}$ samples with the cement according to manufacture's instructions as follow:

Equal amounts of paste $\mathrm{A}$ and $\mathrm{B}$ were dispensed on paper pad and mixed for 20 seconds, applied to both the small and large samples without any ceramic primer. The small samples attached immediately to the center area of the large samples, and 
loaded using the specially constructed loading device (3 kg load) during cementation (Fig.2). The excess resin cement was removed after light cured*for 2 seconds, then light cured for 20 seconds per surface. The curing tip was as close as possible to the specimen.

\section{B. Single bond universal adhesive+RelyX Ulti- mate cement:}

Another five small conventional zirconia (C) samples were bonded to five large $\mathrm{C}$ samples, and five small translucent zirconia $(\mathrm{T})$ samples were bonded to five large $\mathrm{T}$ samples using the cement as follow:

The conditioned surfaces of both square shaped zirconia samples were coated with Single bond universal adhesive for 20 seconds then air dried until the solvent has completely evaporated $(5 \mathrm{sec})$. Cement was mixed, and applied to both the small \& large samples, then the small samples attached immediately to the center area of the large samples and loaded using the same constructed loading device during cementation. The excess resin cement was removed after light cured for 2 seconds, then light cured for 20 seconds per surface.

All specimens were divided into 2 groups according to the type of zirconia and each group was subdivided into 2 subgroups according to type of the cement used as follow:

- Group C $(\mathrm{n}=10)$ : Conventional zirconia specimens

Subgroup CP: Cemented with Panavia F2.0 (5 specimens)

Subgroup CR: Cemented with RelyX Ultimate (5 specimens)
- Group T $(\mathrm{n}=10)$ : Translucent zirconia specimens

Subgroup TP: Cemented with Panavia F2.0 (5 specimens)

Subgroup TR: Cemented with RelyX Ultimate (5 specimens)

All the cemented specimens were thermocycled between $5-55^{\circ} \mathrm{C}$ in water baths for 5000 cycles with 10 seconds dwell time at each bath and transfer time of 5 seconds using a custom made thermocycling machine.

Shear bond strength (SBS) of each specimen evaluated using a computerized universal testing machine. Each specimen was attached to lower jaw of the machine using a simple device. A beveled chisel shaped metallic attached to upper movable compartment of testing machine was used to direct a parallel shearing force as close as possible to interface with a cross head speed of $0.5 \mathrm{~mm} / \mathrm{min}$ (Figure 3).

The failure load was recorded in Newton $(\mathrm{N})$. The shear bond strength values in Mega Pascal (MPa) were calculated for all tested specimens using the following equation:

$$
\tau=\mathrm{F} / \mathrm{A}
$$

Values were presented as mean \pm standard deviation. Statistical analysis was performed using SPSS version 16. Paired t-test was also used to compare between two subgroups. P-value $<0.05$ was considered statistically significant (S) and p-value $\geq 0.05$ was considered statistically nonsignificant (NS).

One extra specimen was prepared from each subgroup using the same previous steps. Cut the specimen to detect the cement/zirconia interface under SEM at different magnifications. 


\section{RESULTS}

1. Shear bond strength (SBS) of conventional zirconia (C) specimens bonded with Panavia F2.0 \& RelyX Ultimate cements (Subgroups CP \& CR):

The results showed a higher mean shear bond strength of the conventional zirconia specimens bonded with RelyX Ultimate cement (15.35 MPa) than that bonded with Panavia F2.0 cement (14.15 $\mathrm{MPa}$ ), and there was no statistically significant difference between two subgroups (P value 0.680), (Table 1 \& Figure 1).

Table (1) Mean shear bond strength (MPa) and standard deviation of conventional zirconia $(C)$ specimens bonded with Panavia F2.0 \& RelyX Ultimate cements (Subgroups $C P$ and $C R$ ):

\begin{tabular}{|c|c|c|c|}
\hline Items & Subgroup CP & Subgroup CR & P-value \\
\hline Mean \pm SD & $14.15 \pm 4.70$ & $15.35 \pm 4.11$ & $\begin{array}{c}0.680 \\
(\mathrm{NS})\end{array}$ \\
\hline
\end{tabular}

NS: indicate insignificant difference $(P>0.05)$.

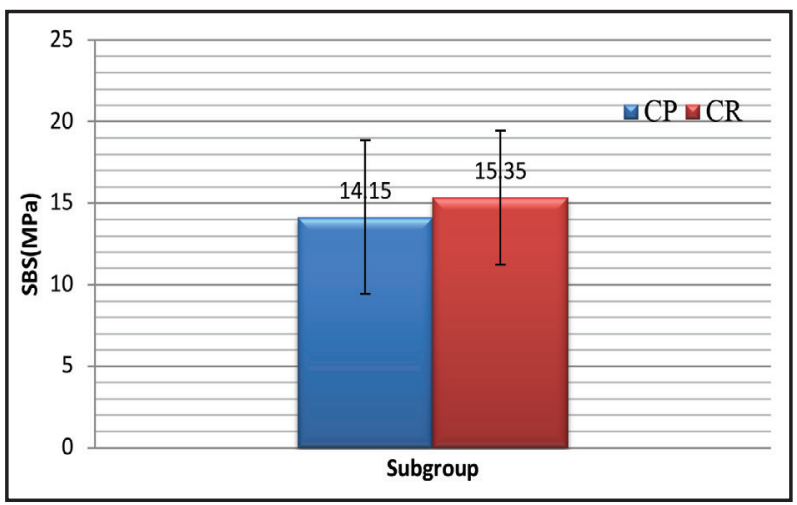

Fig. (1): Mean shear bond strength and standard deviation in MPa of conventional zirconia (C) specimens bonded with Panavia F2.0 \& RelyX Ultimate cements
2. Shear bond strength of translucent zirconia specimens bonded with Panavia F2.0 \& RelyX Ultimate cements (Subgroups TP \& TR):

The results of this study showed a higher mean shear bond strength of the translucent zirconia specimens bonded with RelyX Ultimate cement (18.06 MPa) than that bonded with Panavia F2.0 cement $(15.91 \mathrm{MPa})$, there was no statistically significant difference between both subgroups (P value 0.517 ) (Table $2 \&$ Figure 2).

Table (2) Mean shear bond strength (MPa) and standard deviation of translucent zirconia $(T)$ specimens bonded with Panavia F2.0 \& RelyX Ultimate cements (Subgroups TP \& TR):

\begin{tabular}{|c|c|c|r|}
\hline Items & Subgroup TP & Subgroup TR & P-value \\
\hline Mean \pm SD & $15.91 \pm 4.40$ & $18.06 \pm 5.96$ & $\begin{array}{r}0.517 \\
(\mathrm{NS})\end{array}$ \\
\hline
\end{tabular}

NS: indicate insignificant difference $(\mathrm{P}>0.05)$.

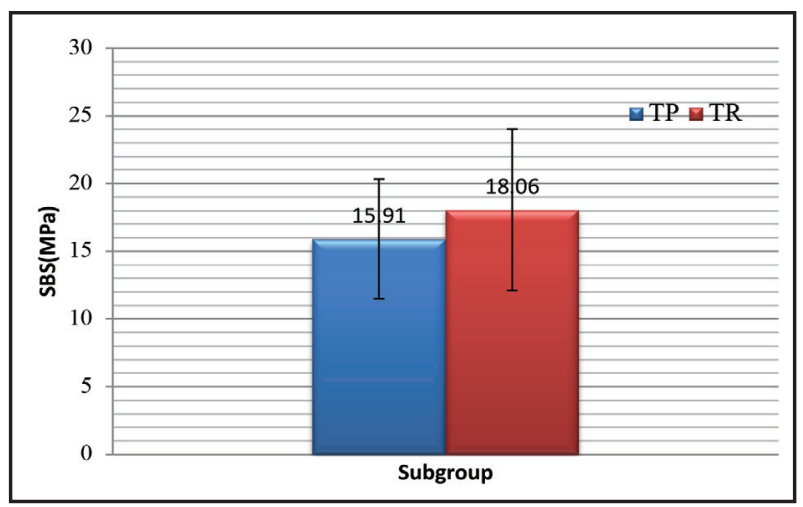

Fig. (2): Mean shear bond strength and standard deviation of translucent zirconia (T) specimens bonded with Panavia F2.0 \& RelyX Ultimate cement 
3. Shear bond strength of the two types of zirconia specimens bonded with Panavia F2.0 cement (Subgroups CP and TP):

Results showed a higher mean shear bond strength of the translucent zirconia specimens bonded with Panavia F2.0 cement (15.91 MPa) than that with the conventional zirconia (14.15 MPa), there was no statistically significant difference (P value 0.557) (Table 3 \& Figure 3).

Table (3): Mean shear bond strength (MPa) and standard deviation of the two types of zirconia specimens bonded with Panavia F2.0 cement (Subgroups CP and TP):

\begin{tabular}{|c|r|r|c|}
\hline Items & Subgroup CP & Subgroup TP & P-value \\
\hline Mean $\pm S D$ & $14.15 \pm 4.70$ & $15.91 \pm 4.40$ & $\begin{array}{c}0.557 \\
(\mathrm{NS})\end{array}$ \\
\hline
\end{tabular}

NS: indicate insignificant difference $(P>0.05)$.

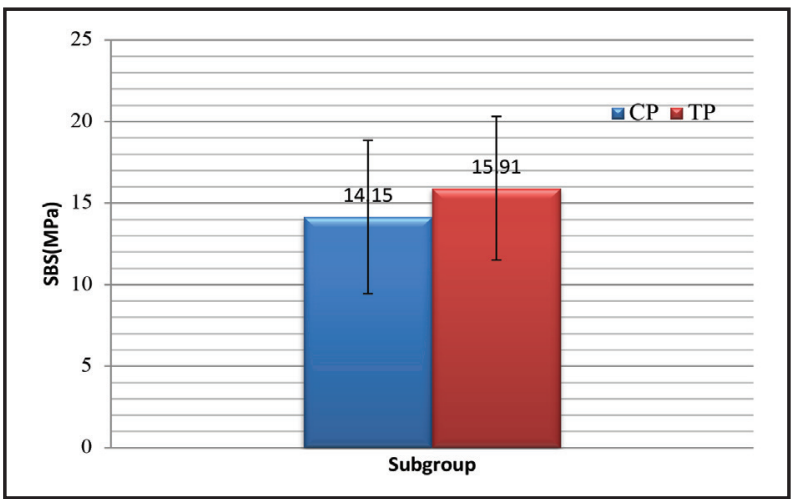

Fig. (3): Mean shear bond strength (MPa) and standard deviation of the two types of zirconia specimens bonded with Panavia F2.0 cement (Subgroups CP and TP)
4. Shear bond strength of the two types of zirconia specimens bonded with RelyX Ultimate cement (Subgroups CR and TR):

Showed a higher mean shear bond strength of the translucent zirconia specimens bonded with RelyX Ultimate cement (18.06 MPa) than that with the conventional zirconia (15.35 MPa), there was no statistically significant difference between two subgroups (P value 0.428) (Table 4 \& Figure 4).

Table (4): Mean shear bond strength (MPa) and standard deviation of the two types of zirconia specimens bonded with RelyX Ultimate cement (Subgroups $C R$ and $T R$ ):

\begin{tabular}{|c|c|c|c|}
\hline Items & Subgroup CR & Subgroup TR & P-value \\
\hline Mean \pm SD & $15.35 \pm 4.11$ & $18.06 \pm 5.96$ & $\begin{array}{c}0.428 \\
(\mathrm{NS})\end{array}$ \\
\hline
\end{tabular}

NS: indicate insignificant difference $(P>0.05)$.

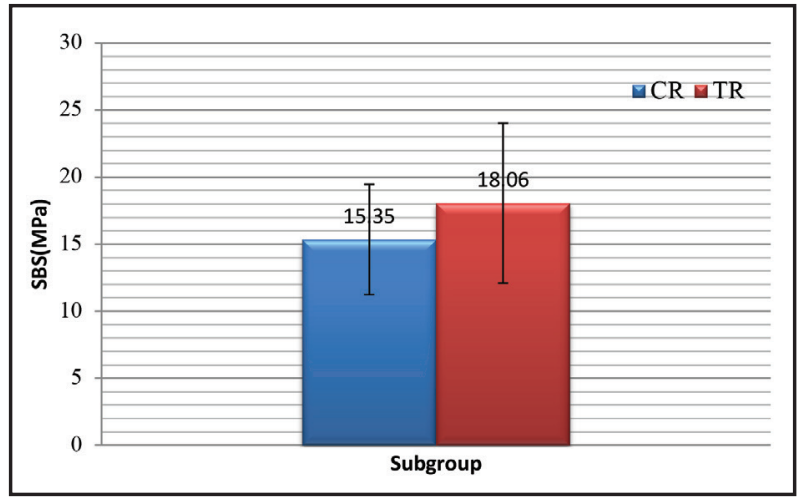

Fig. (4): Mean shear bond strength and standard deviation of the two types of zirconia specimens bonded with RelyX Ultimate cement (Subgroups CR and TR)

\section{Results of the Scanning Electron Microscope:}

Subgroups CR\&TR showed better adaptation than subgroups CP\&TP. While interface contact between translucent zirconia and two types of cement (TP\&TR) is better than that with conventional zirconia (CP\&CR). 


\section{DISCUSSION}

Zirconia used in dental restoration due to its excellent mechanical properties, biocompatibility, low degree of bacterial adhesion, and acceptable optical properties ${ }^{(9)}$. Despite these advantages, zirconia have a major drawback, the chipping of the veneering ceramic ${ }^{(10,11)}$. Monolithic zirconia restorations recently used to overcome chipping problems ${ }^{(12)}$. Bonding properties are important for the adhesive stability of dental restorative materials.

In the present study, ten square shaped large samples from Incoris ZI block and another ten from Incoris TZI block were constructed with dimensions $12 \mathrm{~mm} \times 12 \mathrm{~mm}$. Another ten square shaped small samples were with dimensions $6 \mathrm{~mm} \times 6 \mathrm{~mm}$ from each type of zirconia. The thickness $2 \mathrm{~mm}$ was done to allow chisel tip to direct a parallel force as close as possible to zirconia/cement interface during shear bond strength test. This is in the range of sample dimensions in most previous studies ${ }^{(13,14)}$. All fully sintered large samples were embedded in self cured acrylic resin blocks in a rectangular plastic mould with only one side was exposed and would be parallel to the edge of resin blocks and to long axis of the chisel of the universal testing machine during the shear test.The specimens design of ceramic to ceramic was adopted from several studies in which two ceramic samples were bonded together ${ }^{(14-17)}$.

Samples in this study were air-abraded by $110 \mu \mathrm{m} \mathrm{AL}_{2} \mathrm{O}_{3}$ particles under 2.5 bar pressure for $15 \mathrm{sec}$ from distance of $10 \mathrm{~mm}$. This causes insignificant damage to the surface and achieve superior bond $^{(18-20)}$. Distance between the nozzle and the surface to be blasted was selected to be $10 \mathrm{~mm}$ in the present study because this provide sufficient surface roughness ${ }^{(19,21)}$. Duration of sandblasting was selected to be 15 seconds because sandblasting for a longer time may cause sharp margins that act as stress points which may lead to formation and propagation of cracks ${ }^{(22,23)}$.
The cementation procedures is essential for success of restorations ${ }^{(24)}$. Air abrasion alone may not be enough to achieve satisfactory bonding between resin cement and zirconia due to the lack of chemical bonding between the methacrylate monomer of the resin cement and zirconia. So MPD phosphate monomer was recommended to be used to achieve durable bond with zirconia ${ }^{(8,24,25,26)}$, as MDP is a functional group with a long organic hydrophobic chain molecule with two ends. One end has vinyl groups that react with the monomers of the resin cement when polymerized and the other end, hydrophilic phosphate ester groups bond strongly with oxide layers ${ }^{(27,28)}$.

Cementation of specimens in the current study was conducted using the investigated resin cements: Panavia F 2.0 (MDP-containing cement), and Single Bond Universal Adhesive/RelyX Ultimate (MDP-containing primer/ MDP-free cement). The specimens were light cure, kept under pressure until complete setting of the cements to prevent creation of cracks within the cement layer. Bonding procedures was standardized by applying a load of 3 $\mathrm{Kg}$ on all specimens to exclude human variations that could happen during cementation. This load value was recommended by Rinke et $\mathrm{al}^{(29)}$ and Gorten and Probester ${ }^{(30)}$ to avoid the risk of damage of the bond between the zirconia samples. Specimens were immersed in water bath for 5000 cycles with 10 seconds dwell time at each bath. As short dwelling time will probably not allow the specimens to reach quickly the temperature of the water bath and this could easily lead to inadequate exposure as reported by Walls et $\mathrm{al}^{(31)}$.

Many studies on bond strengths of adhesive resin cements used crosshead speeds which varied between 0.2 to $1 \mathrm{~mm} / \mathrm{min}$ but the vast majorities of these studies adopted 0.5 or $1 \mathrm{~mm} / \mathrm{min}{ }^{(32-34)}$. The crosshead speed used in this study was $(0.5 \mathrm{~mm} / \mathrm{min})$ 
as it was considered to be at the middle of the range of crosshead speeds and provided more reliable shear bond strength values.

SEM was used to view cement/zirconia interface, showed that Panavia F 2.0 has more gaps in the cement/zirconia interface with both types of zirconia surfaces than Single bond universal adhesive with RelyX Ultimate. This might be due to the poorer spreadability of cement emerging from its higher viscosity that hindered its flow and adhesion to zirconia ${ }^{(35)}$.

In the present study, two independent variables could affect the bond strength: the type of zirconia and cement system used. Results showed that there was no statistically significant difference between the shear bond strength of conventional \& translucent zirconia cemented by both cements (CP 14.15MPa, TP 15.91 MPa) (CR 15.35 MPa, TR 18.06 MPa). This might be due to the close similarity in chemical compositions between the two types of zirconia ${ }^{(36,37)}$, and the presence of the functional monomer MDP in the Single bond universal adhesive applied before RelyX Ultimate cement, and in Panavia F2.0 cement ${ }^{(38,39)}$. This could be in aggrement with Shaymaa E Elsaka ${ }^{(40)}$ who concluded that the bond strength was significantly affected by the surface treatment and the type of resin cement, but not by the type of zirconia .

However, SBS values was slightly higher in translucent zirconia (TP 15.91MPa, TR 18.06 MPa) than in conventional zirconia (CP 14.15MPa, CR 15.35MPa). This could be related to the proper transmittance of the light through the translucent zirconia that could increase the polymerization of the resin cement ${ }^{(41,42)}$. Translucency of zirconia is an important parameter in matching the appearance of the natural tooth and for polymerization of resin cement used for cementation of these restorations. Light transmission is inversely correlated with the thickness of restoration and the light scattering rate of ceramic ${ }^{(43)}$. Resin cement is photo-activated through a restoration, part of the light is absorbed and part is reflected on the surface of the restoration, while the part that reaches the cement is the one that is transmitted through the restoration. This depends on the optical characteristics of the restorative material ${ }^{(44)}$. This justification is in line with previous studies ${ }^{(42,45)}$ which reported that traditional zirconia is a less translucent material, and the transmittance of the curing light through it may not always be adequate to ensure a proper degree of conversion of the resin cement. In order to overcome the limits towards bonding procedures, translucent zirconia improved esthetic properties and provide better transmittance of light through it. Visuttiwattanakorn ${ }^{(14)}$ evaluated light transmission through 6 types of zirconia ceramics and showed that inCoris TZI was more translucent than other types of zirconia. Another reason for the lower shear strength produced between conventional zirconia and resin cement is that the oxides content in Incoris TZI are more than that in Incoris ZI ${ }^{(36,37)}$. MDP is an acidic phosphate monomer which is originally designed to bond to metal oxides and its use has been extended to zirconia. Zirconia surface is easily covered with a passive oxide layer which makes zirconia similar to metals ${ }^{(6)}$. Shear bond strength of zirconia specimens bonded with RelyX Ultimate in combination with Single bond universal adhesive was slightly higher than those bonded with Panavia F 2.0. The reason for that might be due to the higher viscosity of Panavia F2.0 cement than Single bond universal adhesive and this proved with SEM. The adhesive used with RelyX Ultimate penetrate more into irregularities created by air abrasion and could promote better surface wettability ${ }^{(46,49)}$. This was in agreement with Rohr N. ${ }^{(47)}$ as he reported in a study, that MDP in primer showed significant difference than that of cement in improving bond strength of zirconia by increased wettability. 
The results of the current study is in accordance with Wolfart et $\mathrm{al}^{(8)}$, Nothdurft et $\mathrm{al}^{(48)}$, Taniş et $\mathrm{al}^{(21)}$, Visuttiwattanakorn et $\mathrm{al}^{(14)}$, Al Jeaidi et $\mathrm{al}^{(49)}$.

In contrast with Kulunk et $\mathrm{al}^{(13)}$ who showed that shear bond strength after air abrasion with $110 \mu \mathrm{m}$ $\mathrm{Al}_{2} \mathrm{O}_{3}$ particles of zirconia to panavia $\mathrm{F} 2.0$ was $30.5 \mathrm{MPa}$, this might because using another type of zirconia (ICEZircon Translucent; Zirconzahn). Also the results of bond strength of Panavia F 2.0 to zirconia were in contrast with Ozcan et $\mathrm{al}^{(50)}$ who reported no adhesion of Panavia F 2.0 to zirconia after thermocycling, this difference might be attributed to the difference in methodology, like using air-particle abrasion with $50 \mathrm{um} \mathrm{AL}_{2} \mathrm{O}_{3}$, which might lead to less surface roughness.

\section{CONCLUSION}

Within the limitations and conditions of this invitro study, it could be concluded that :

1. Adhesion between MDP containing resin cements or primer to zirconia is not affected by the type of zirconia (conventional, translucent).

2. There is no difference in the bond strength between Panavia F2.0 and Single bond universal adhesive+RelyX Ultimate resin cements to zirconia.

- Acknowledgment: I would like to express my sincere gratitude and deepest appreciation to $\mathrm{Dr} /$ Mosaad Aly El-Gabrouny \& Dr/ Amr Abd El-Aziz Shebl for all helps, bright thoughts, sincere advice, continuous supervision for all lab test of the article and gentle support.

- Conflict of interest: Authors deny any conflict of interest

\section{Conflict of interest}

- Authors deny any conflict of interest.

\section{REFERENCES}

1. Kelly JR, Nishimura I, and Campbell SD. Ceramics in dentistry:historical roots and current perspectives. Journal of Prosthetic Dentistry. 1996; 75:18-32.

2. Wang F, Takahashi H, and Iwasaki N. Translucency of dental ceramics with different thickness. J Prosthet Dent 2013; 110:14-20.

3. Blatz MB. Long term clinical success of all-ceramic posterior restorations. Quintessence Int. 2002; 33:415-426.

4. Harianawala HH, Kheur MG, Apte SK, Kale BB, Sethi TS, and Kheur SM. Comparative analysis of transmittance for different types of commercially available zirconia and lithium disilicate materials. J Adv Prosthodont 2014; 6:456-461.

5. Nakamura K, Harada A, Inagaki R, Kanno T, Niwano Y, Milleding P, and Örtengren U. Fracture resistance of monolithic zirconia molar crowns with reduced thickness. Acta Odontol Scand. 2015; 73:602-608.

6. Blatz MB, Sadan A, Martin J, Lang B. In vitro evaluation of shear bond strengths of resin to densely-sintered high purity zirconium-oxide ceramic after long-term storage and thermal cycling.J Prosth Dent.2004; 91:356-362.

7. Blatz MB, Sadan A, Kern M. Resin-ceramic bonding: a review of the literature. J Prosthet Dent. 2003; 89:268-274.

8. Wolfart M, Lehmann F, Wolfart S, Kern M. Durability of the resin bond strength to zirconia ceramic after using different surface conditioning methods. Dent Mater. 2007;23:45-50.

9. Denry I, Kelly JR. State of art of zirconia for dental applications. Dent Mater 2008; 24:299-307.

10. Sax C, Hämmerle CH, and Sailer I. 10-year clinical outcomes of fixed dental prostheses with zirconia frameworks. Int J Comput Dent. 2011;14:183-202.

11. Kou W, Molin M, SjoGren G. Surface roughness of five different dental ceramic core materials after grinding and polishing. J Oral Rehab 2006 ;33: 117-124.

12. Rojas-Vizcaya F. Full zirconia fixed detachable implantretained restorations manufactured from monolithic zirconia: clinical report after two years in service. J Prosthodont. 2011;20:570-576. 
13. Kulunk S, Kulunk T, Ural C, Kurt M, and Baba S. Effect of air abrasion particles on the bond strength of adhesive resin cement to zirconia core. Acta Odontologica Scandinavica , 2011; 69: 88-94.

14. Visuttiwattanakorn P, Rithy N, Suputtamonkol K, and Kanchanavasita W. Shear bond strength of Zirconia to different adhesive resin cements. M Dent J. 2015;35:127-136.

15. Yoshida K, Tsuo Y, and Atsuta M. Bonding of dual-cured resin cement to zirconia ceramic using phosphate acid ester monomer and zirconate coupler. J Biomed Mater Res B Appl Biomater 2006 ;77: 28-33.

16. Uo M, Sjogren G, Sundh A, Goto M, Watari F, and Bergman M. Effect of surface condition of dental zirconia ceramic (Denzir) on bonding. Dent Mater J 2006; 25:626-631.

17. Lin J, Shinya A, and Gomi H. Effect of self-adhesive resin cement and tribochemical treatment on bond strength to zirconia. Int J Oral Sci 2010; 2:28-34.

18. Lee HJ, Ryu JJ, Shin SW, Suh KW. Effect of surface treatment methods on the shear bond strength of resin cement to zirconia ceramic. J Korean Acad Prosthodont. 2007; 45:743-752.

19. Kim MJ, Kim YK, Kim KH, Kwon TY. Shear bond strengths of various luting cements to zirconia ceramic: surface chemical aspects. J Dent. 2011 ; 39:795-803.

20. Juntavee N, Apa Juntavee, Yothajun T. Effects of different surface treatments on bond strength between resin cements and zirconia ceramics. Oper Dent. 2014; 39:118-27.

21. Merve Çakirbay Taniş, Canan Akay, and Duygu Karakiş. Resin cementation of zirconia ceramics with different bonding agents. Biotechnol Biotechnol Equip. 2015; 29: 363-367.

22. Sarmento HR, Campos F, Sousa RS, Machado JP, Souza RO, Bottino MA, and Ozcan M. Influence of air-particle deposition protocols on the surface topography and adhesion of resin cement to zirconia. Acta Odontol Scand. 2014;72:346-353.

23. Zeighami S, Gheidari A, Mahgoli H, Rohanian A, and Ghodsi S. Effect of sandblasting angle and distance on biaxial flexural strength of zirconia-based ceramics. J Contemp Dent Pract. 2017 Jun 1;18:443-447.
24. Donovan TE. Factors essential for successful all-ceramic restorations. J Am Dent Assoc 2008;139:14-18.

25. Blatz MB, Phark JH, Ozer F, Mante FK, Saleh N, Bergler $\mathrm{M}$, Sadan A. In vitro comparative bond strength of contemporary self-adhesive resin cements to zirconium oxide ceramic with and without air-particle abrasion. Clin Oral Investig. 2010;14:187-192.

26. Qeblawi DM, Muñoz CA, Brewer JD, Monaco EA Jr. The effect of zirconia surface treatment on flexural strength and shear bond strength to a resin cement. J Prosthet Dent. $2010 ; 103: 210-220$.

27. Lehmann F, Kern M. Durability of resin bonding to zirconia ceramic using different primers. J Adhes Dent. 2009; 11:479-483.

28. Liang Chen and Byoung In Suh. Bonding of resin materials to all-ceramics: A Review. Current Research in Dentistry 2012; 3:7-17.

29. Rinke S, Huls A, and Jahn L. Marginal accuracy and fracture strength of conventional and copy-milled all-ceramic crowns. Int J Prosthodont 1995; 8:45-52.

30. Groten M., and Probester L. The influence of different cementation modes on the fracture resistance of feldspathic ceramic crowns. Int J Prosthodont 1997; 10:169-177.

31. Walls AW, McCabe, and Murray JJ. The bond strength of composite laminate veneers. J Dent Res 1985; 64:12611264.

32. Tanaka R, Fujishima A, Shibata Y, Manabe A, Miyazaki T. Cooperation of phosphate monomer and silica modification on zirconia. J Dent Res 2008; 87:666-670.

33. De Munk J, Vargas M, Van Landuyt K, Hikita K, Lambrechts P, and Van Meerbeek B. Bonding of an autoadhesive luting material to enamel and dentin. Dent Mater $2004 ; 20: 963-971$.

34. Piwowarczyk A, Lauer HC, and Sorensen JA. In vitro shear bond strength of cementing agents to fixed prosthodontics restorative materials. J Prosth Dent 2004 ;92:265-273.

35. J.Zhao, X.Wang, W.Si, Z.Shen. Effect of resin cement selection on the microtensile bond strength of adhesively veneered 3Y-TZP. ActaOdontol. 2013; 71:1105-11.

36. https:// media. dentalcompare. com/m/25/Downloads/ inCoris \%20ZI\% 20 Zirconium \% 20Oxide\%20Ceramic\%20Blocks $\% 20$ for $\%$ 20inLab\% 20 Processing\% 20Instructions.pdf 
37. https://www.dentsplysirona.com/content/dam/flagship/ en-us/products/cad-cam/cad-cam-for-laboratories/inlabmaterials-downloads/11-2011-inCoris-TZI-ProcessingInstructions.pdf

38. www.panavia-dental.eu/en/fag.com.

39. https://multimedia.3m.com/mws/media/1278632O/3msingle-bond-universal- adhesive-faq.pdf.

40. Shaymaa E Elsaka. Influence of Surface Treatments on the Bond Strength of Resin Cements to Monolithic Zirconia.J Adhes Dent 2016; 18:387-395.

41. Turp V, Sen D, Poyrazoglu E, Tuncelli B, and Goller G. Influence of zirconia base and shade difference on polymerization efficiency of dual-cure resin cement. J Prosthodont 2011; 20:361-365.

42. Ilie N, and Stawarczyk B. Quantification of the amount of blue light passing through monolithic zirconia with respect to thickness and polymerization conditions. J Prosthet Dent 2015; 113:114-121.

43. Burke FJ, Fleming GJ, Nathanson D, Marquis PM. Are adhesive technologies needed to support Ceramics? An assessment of the current evidence. J Adhes Dent 2002; 4: 7-22.

44. Ilie N, Stawarczyk B. Quantification of the amount of light passing through zirconia: the effect of material shade, thickness, and curing conditions. J Dent 2014; 42:684-690.

45. Kurtulmus-Yilmaz S, and Ulusoy M. Comparison of the translucency of shaded zirconia all-ceramic systems. J Adv Prosthodont 2014; 6:415-422.

46. Zhao L, Jian YT, Wang XD, and Zhao K. Bond strength of primer/ cement systems to zirconia subjected to artificial aging. J Prosthet Dent. 2016; 116:790-796.

47. Rohr N, Brunner S, Märtin S, and Fischer J . Influence of cement type and ceramic primer on retention of polymerinfiltrated ceramic crowns to a one-piece zirconia implant. J Prosthet Dent. 2018 ;119:138-145.

48. Nothdurft FP, Motter PJ, Pospiech PR. Effect of surface treatment on the initial bond strength of different luting cements to zirconium oxide ceramic. Clin Oral Investig. 2009;13 :229-235.

49. Al Jeaidi Z, Alqahtani M , Awad M, Rodrigues F, and Alrahlah A. Bond strength of universal adhesives to air-abraded zirconia ceramics. Journal of Oral Science 2017;59: 565-570.

50. Ozcan M, Kerkdijk S, and Valandro LF. Comparison of resin cement adhesion to Y-TZP ceramic following manufacturer's instructions of the cements only. Clin Oral Invest $2008 ; 2: 79-82$. 